\title{
On the relation between decision rules and perceptual representation in multidimensional perceptual categorization
}

\author{
W. TODD MADDOX and SERGEI V. BOGDANOV \\ University of Texas, Austin, Texas
}

\begin{abstract}
This article examines the relation between changing categorization decision rules and the nature of the underlying perceptual representation. Observers completed a matching task that required them to adjust the length and orientation of a single line stimulus until they perceived it to "match" a second line stimulus (Alfonso-Reese, 1996, 1997). The same observers then completed four categorization tasks with the same stimuli. Data from the matching task were used to estimate a perceptual representation for each stimulus and observer. Three hypotheses regarding potential interactions between categorization decision rules and perceptual representation were examined. One assumed that there was no interaction between decision rules and perceptual representation. The second assumed that linear categorization rules affect the perceptual representation differently from nonlinear categorization rules. The third assumed that dimensional integration rules affected the perceptual representation differently from decision rules that require the observer to set a criterion along one stimulus dimension while ignoring the other; this is referred to as decisional selective attention. The results suggested that (1) the matching task perceptual representation provided a good account of the categorization data, (2) decisional selective attention affected the perceptual representation differently from decisional integration, and (3) decisional selective attention generally decreased the perceptual variability along the attended dimension.
\end{abstract}

Categorization is a primary component of many behaviors of all organisms (Alcock, 1989). In general, each category exemplar can be decomposed into a set of values along multiple basic stimulus dimensions, each of which is continuous rather than binary valued (e.g., Ashby, 1992a; Ashby \& Maddox, 1998). For example, category exemplars may vary along the dimensions of size, length, orientation, color, location, frequency, and amplitude. Because each dimension is processed differently by the perceptual system, it is important to understand the nature of the perceptual representation and how it affects categorization performance (Alfonso-Reese, 1997; Ashby \& Lee, 1991; Maddox \& Ashby, 1996; Nosofsky, 1986). Conversely, recent research suggests that the act of categorization might alter the structure of the perceptual representation (e.g., Ashby \& Lee, 1991; Goldstone, 1994; Maddox \& Ashby, 1996; Nosofsky, 1986). In particular, different categorization rules might alter the structure of the perceptual representation in different ways. This article addresses both of these issues within a single ex-

This research was supported in part by National Science Foundation Grant SBR-9796206 and NIH Grant R01 MH59196. We thank Helena Kadlec, Robin Thomas, and an anonymous reviewer for helpful comments on an earlier draft of this manuscript. We also thank Greg Ashby, Leslie Cohen, Randy Diehl, Bill Geisler, David Gilden, Art Markman, and Tom Thornton for discussions that influenced this work. Correspondence should be addressed to W. T. Maddox, Department of Psychology, Mezes Hall 330, University of Texas, Austin, TX 78712 (e-mail: maddox@psy.utexas.edu). perimental framework. Each observer completed two tasks using the same set of line stimuli. The first task, a matching task (to be described shortly; Alfonso-Reese, 1996, 1997), was used to derive an initial perceptual representation for the set of line stimuli. The second task was a categorization task that consisted of four separate conditions. The categorization conditions were modeled after those from an influential study conducted by Nosofsky (1986). The effects of perceptual representation on categorization performance were examined by evaluating the ability of the perceptual representation (derived from the matching task) to account quantitatively for the categorization data from each of the four conditions. For completeness, two other popular perceptual representations were also examined (Ashby \& Lee, 1991; Maddox \& Ashby, 1993, 1996). The effects of categorization decision rules on the perceptual representation were examined by postulating changes in the perceptual representation that are specific to various decision rules and by evaluating their ability to account quantitatively for the categorization data. Of particular interest was to quantify the effects of decisional selective attention rules on the nature of the perceptual representation-that is, decision rules that require the observer to set a criterion on one stimulus dimension while ignoring the other stimulus dimension. Performance in decisional selective attention tasks is often quite different from performance in decisional integration tasks, which require the observer to integrate information across dimensions when generating a response. A comparison of decisional selective attention 
and decisional integration rules was a major focus of Nosofsky's (1986) study. Using an exemplar-similarity approach, Nosofsky (1986) found that the similarity relations among items in the multidimensional (MDS) psychological space differed qualitatively across the two types of decision rules. Although an important finding in its own right, one weakness of the exemplar-similarity approach is that it does not separate perceptual processes from decisional processes. Thus, it is unclear whether the "selective attention" effect observed by Nosofsky (1986) is due to changes in perceptual processes, decisional processes, or both. A major focus of the present research is to determine the locus of this "selective attention" effect. To achieve this goal we utilized a different theoretical approach, decision bound theory, which postulates separate and uniquely identifiable perceptual and decisional processes.

The following two (second and third) sections of the article briefly review the relevant literature and introduce decision bound theory. The fourth section details the methods, and the fifth section reports the results and theoretical analyses. Finally, we close with some general comments.

\section{Effects of Perceptual Representation on Categorization Performance}

It is clear from psychophysics research that perceptual processing is probabilistic; that is, repeated presentations of the same stimulus yield different perceptual effects (e.g., Ashby \& Townsend, 1986; Geisler, 1989; Geisler \& Albrecht, 1997; Geisler \& Chou, 1996; Green \& Swets, 1967). This is termed perceptual noise. Over trials, a single multidimensional stimulus can be represented perceptually by a multivariate probability distribution. Despite the importance of noise in perceptual processing, few categorization theories incorporate explicitly perceptual noise into their framework. Many theories assume that there is no perceptual noise and that each category exemplar can be represented by a point in some MDS psychological space (e.g., Nosofsky, 1986) or by a set of feature values (e.g., Medin \& Schaffer, 1978). Although this assumption may be reasonable with some artificial stimuli, it is clearly incorrect in terms of the dimensions of real-world objects, which show noise to be a fundamental component of all perceptual systems. A notable exception is decision bound theory (also called general recognition theory), which is a generalization of signal detection theory to multidimensional stimuli (e.g., Ashby, 1992a; Ashby \& Lee, 1991; Ashby \& Maddox, 1993, 1994; Ashby \& Perrin, 1988; Ashby \& Townsend, 1986; Kadlec \& Hicks, 1998; Maddox \& Ashby, 1993; Thomas, 1995). For a two-dimensional stimulus, a bivariate normal distribution is assumed to describe the set of percepts. A bivariate normal distribution is described by a mean and variance along each dimension, as well as a covariance term, $\mu_{x}, \mu_{v}, \sigma_{x}^{2}, \sigma_{y}^{2}, \operatorname{cov}_{x y}$, where the subscripts $x$ and $y$ denote dimensions $x$ and $y$. Figure 1 depicts hypothetical equal likelihood contours for four

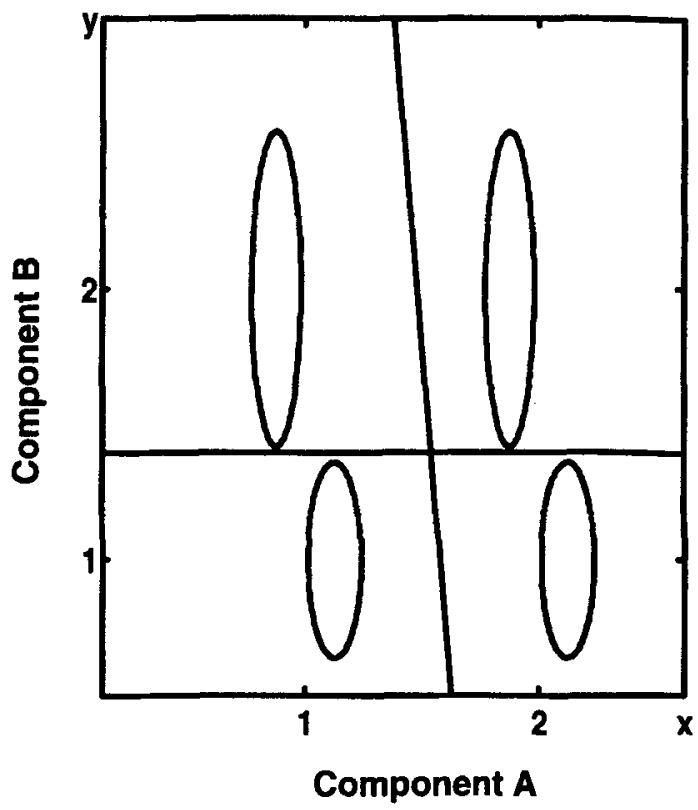

Figure 1. Hypothetical response regions and perceptual space for four stimuli constructed from the factorial combination of two levels along two stimulus components. Perceptual and decisional separability are satisfied along Component $B$ and are violated along Component $\mathbf{A}$.

stimuli constructed from the factorial combination of two levels along two dimensions $x$ and $y$. With bivariate normal distributions, the equal likelihood contours are always circular or elliptical. When the major and minor axes of the contour are parallel to the coordinate axes, the covariance (correlation) is zero. All four distributions in Figure 1 have zero correlations. A positive slope for the major axis implies a positive covariance (correlation), and a negative slope implies a negative covariance. The greater the variance along a dimension, the wider the contour in that direction. Notice that there is greater variance along dimension $y$ for all four stimuli.

In decision bound theory, the experienced observer learns to divide the perceptual space into response regions and assigns a categorization response to each region. The partition between separate response regions is called the decision bound. Within the framework of decision bound theory, the perceptual representation parameters are separate, and in most cases are uniquely identifiable, from the parameters that denote the decision bound. This is an important property of the theory because our goal is to tease apart the separate influences of perceptual and decisional processes.

In contrast, in exemplar models, such as Nosofsky's (1986) generalized context model, perceptual and decisional factors affect the nature of the psychological space, often operating on the same parameters. Thus, the perceptual representation parameters are not separate, and iden- 
tifiable, from the parameters that define the decision process (see Maddox \& Ashby, 1998, and Nosofsky, 1998, for a discussion). For example, the attention weight parameter, $w$, in the generalized context model modifies the similarity relations among items in the psychological space and thus has a strong effect on the equal similarity contour that separates the psychological space into two regions- one in which the probability of responding " $\mathrm{A}$ " is greater than .5 and the other in which the probability is less than .5. However, Ashby and Perrin (1988; see also Maddox \& Ashby, 1998) showed that the attention weight parameter is directly related to the perceptual variance parameters in a simple version of decision bound theory in which the perceptual covariance matrices are identical across stimuli and in which perceptual independence is satisfied, constraints that were not satisfied in the present study. Thus, the attention weight parameter is also directly related to the perceptual form of attention postulated in decision bound theory.

One approach to studying the effects of perceptual representation on categorization has been to estimate the perceptual representation and decision rule parameters simultaneously from data collected in several categorization conditions (e.g., Maddox \& Ashby, 1993). One weakness of this approach is that often too few degrees of freedom are available for testing general perceptual representations, and so simplifying assumptions are usually made. An alternative approach is to estimate the perceptual distribution parameters from data collected in one task, such as an identification task, and then to use the resulting perceptual representation to predict quantitatively performance in a series of categorization tasks using the same stimuli and observers. The advantage of this approach is that more general perceptual representations can be examined. Ashby and Lee (1991) took this approach and found that the perceptual representation estimated from identification provided a good description of performance across four different categorization conditions (see also Kadlec \& Hicks, 1998; Kingston \& Macmillan, 1995; Kingston, Macmillan, Dickey, Thorburn, \& Bartels, 1997; Macmillan \& Kingston, 1995; Macmillan \& Ornstein, 1998; Maddox \& Ashby, 1996; Nosofsky, 1986; Wickens \& Olzak, 1992).

\section{Effects of Categorization Decision Rule on Perceptual Representation}

A growing body of research suggests that the act of categorization might alter the structure of the perceptual representation (e.g., Ashby \& Lee, 1991; Goldstone, 1994; Maddox \& Ashby, 1996; but see Kadlec \& Hicks, 1998). For example, Ashby and Lee (1991; see also Maddox \& Ashby, 1996) found that a perceptual representation that satisfied perceptual separability provided a better account of the categorization data than the perceptual representation estimated from the identification data. ${ }^{1}$ In other words, the perceptual representation that best described the categorization data was different from the perceptual representation that best described the identification data. The implication is that the act of categorization changed the structure of the perceptual representation. Ashby and Lee (1991) did not examine the possibility that the perceptual representation might be altered differently by different categorization decision rules. They assumed that the perceptual representation was the same across all categorization conditions. We refer to this as categorization condition invariance because the representation is assumed to be invariant across categorization conditions. We test this categorization condition invariance hypothesis in the present study.

The hypothesis that categorization decision rules alter perceptual processes is supported by several results from the neuroscience literature. First, single-cell recording work in the monkey suggests that the receptive fields and firing rates of cells change when the task demands change. In particular, there is evidence that the sensitivity of certain cells increase when the task requires decisional selective attention to a single relevant aspect of the stimulus (e.g., Desimone \& Ungerleider, 1989; Moran \& Desimone, 1985; Spitzer, Desimone, \& Moran, 1988). Second, there is widespread agreement that human attention is subserved by separate subsystems. Specifically, perceptual (i.e., visual) attention is mediated by a posterior system that includes visual cortex, much of posterior parietal cortex, the pulvinar, and the superior colliculus. Executive (i.e., conscious) attention is thought to be mediated by an anterior system that includes anterior cingulate, prefrontal cortex, and perhaps the basal ganglia and pulvinar (Ashby, Alfonso-Reese, Turken, \& Waldron, 1998; Desimone \& Duncan, 1995; Goldman-Rakic, 1995; LaBerge, 1997; Olshausen, Anderson, \& Van Essen, 1993; Posner \& Petersen, 1990). Maddox, Ashby, and Waldron (1999) tested empirically the hypothesis that these systems are functionally independent. Maddox et al. (1999) had 4 collegeage observers participate in a categorization task that required the executive attention system to set a decision criterion along one stimulus dimension while ignoring another. On $80 \%$ of the trials the perceptual attention system was focused on the relevant stimulus dimension, whereas on the remaining trials the perceptual attention system was focused on the irrelevant dimension. Maddox et al. (1999) found support for the independence hypothesis but also found evidence that the executive decisional system affected the perceptual representation. Taken together, these results suggest that categorization decision rules can affect the perceptual representation and that this is likely when the categorization decision rule requires decisional selective attention to one aspect of the stimulus.

\section{MATCHING-CATEGORIZATION EXPERIMENT}

The primary goal of the present research was to investigate potential interactions between the categorization decision rule and the nature of the perceptual representation. Our approach was to have observers complete a matching task (referred to as the "noise measurement" 
technique by its developer Alfonso-Reese, 1996, 1997) that would be used to estimate a perceptual representation and to have the same observers complete several different categorization tasks using the same stimuli. ${ }^{2}$ On each trial of the matching task, the observer is presented with two stimuli and the task is to adjust one of the stimuli until it "matches" the other. We collected a large body of data from the matching task and then used these data to estimate the parameters of a perceptual representation for each stimulus. The categorization data, specifically the observed categorization response probabilities for each stimulus, were then predicted quantitatively from the estimated perceptual representation. For completeness, two other perceptual representations were also examined. One assumed perceptual separability (Ashby \& Lee, 1991; Maddox \& Ashby, 1996), and the other assumed stimulus invariance - that is, that the perceptual covariance matrix for each stimulus is identical (Maddox \& Ashby, 1993). Three categorization condition invariance hypotheses were examined. One assumed that the perceptual representation was not differentially affected by different categorization rules (i.e., that categorization condition invariance was satisfied). The second and third hypotheses each instantiated a specific violation of categorization condition invariance.

\section{Method}

\section{Observers}

Three observers participated in the experiment. Observer 1 was S.V.B. Observers 2 and 3 were female college students from Arizona State University. All 3 observers had prior experience with these types of experiments and stimuli.

\section{Stimuli}

Matching. The stimulus set consisted of 12 lines selected from a set of 16 lines that were constructed from a factorial combination of 4 line lengths with 4 line orientations (see Figure $2 a$ ). Previous research suggests that length and orientation are separable (e.g., Garner, 1974; Garner \& Felfoldy, 1970; Shepard, 1964; but see Ashby \& Lee, 1991; Ashby \& Maddox, 1990; Maddox, 1992). The four line lengths were $100,112,124$, and 136 pixels. The four line orientations were $864,1.021,1.178$, and 1.335 radians from horizontal. Observers were seated approximately $40 \mathrm{in}$. from the computer screen, and each stimulus subtended a visual angle of approximately $1^{\circ}$. The stimuli were computer generated and displayed on a noninterlaced VGA monitor with $1024 \times 768$ resolution in a dimly lit room. Each line was presented in white on a black background. ${ }^{3}$

Categorization. The stimulus ensemble for each categorization condition consisted of 8 of the 12 matching task stimuli. The response mappings for the criss-cross, interior-exterior, linear integration, and decisional selective attention conditions are depicted in Figure 2 . These conditions were modeled after those used by Nosofsky (1986).

\section{Procedure}

Matching. On each trial, 1 of the 12 stimuli was selected at random with equal probability. This served as the standard stimulus on that trial. The center of the standard stimulus was presented 150 pixels to the left of the horizontal center of the computer screen and 10 pixels below the vertical center of the screen. The center of the comparison stimulus was presented 150 pixels to the right of the horizontal center of the computer screen and 10 pixels above the vertical center of the screen. The stimuli were offset vertically to minimize the possibility that the observer would use a matching strategy based solely on the endpoints of the stimuli. The length and orientation of the comparison stimulus were determined as follows. First, a length was selected at random from a uniform ( $-50,50$ pixel) distribution and was added to the standard stimulus length. Second, an orientation was selected at random from a uniform $(-.157, .157$ radian) distribution and was added to the standard stimulus orientation. These values were used to construct the comparison stimulus. The observer's task was to adjust the length and orientation of the comparison stimulus until it matched the standard stimulus. Four keys on the keyboard were used to control the adjustment. Two of the keys were used to adjust the length (longer or shorter), and two keys were used to adjust the orientation (steeper or shallower). Each keypress led to a small change in the length or orientation of the comparison stimulus. Small adjustment amounts were chosen so that each unit of change would be imperceptible to the observer. Once the observer perceived the comparison and standard stimuli to match, he/she pressed another key, and the next trial was initiated. Observers were told "to be as accurate as possible." Each session consisted of four blocks of 30 trials with breaks in between each block.

Categorization. After completing the matching task, each observer completed two $1-\mathrm{h}$ sessions in each of four categorization conditions. The order of the conditions was randomized across observers. The condition orders were as follows. Observer 1 : interiorexterior, decisional selective attention, linear integration, criss-cross. Observer 2: criss-cross, decisional selective attention, interiorexterior, linear integration. Observer 3 : decisional selective attention, linear integration, criss-cross, interior-exterior. Each session consisted of four blocks of 200 trials for a total of 800 trials per session. Within each 200-trial block, each of the eight stimuli was presented 25 times. The stimulus sequences were randomized within blocks. The first session of each condition was considered practice and was excluded from all subsequent analysis.

In each condition the observers were instructed that there were two categories of line stimuli and that their task was to categorize each stimulus into one of the two categories by pressing the appropriate key. Accuracy was emphasized over speed of responding. The timing of each trial was as follows. At the start of each trial, a single line stimulus was presented (centered) on the screen for $300 \mathrm{msec}$, followed by a pattern mask. Once the observer responded, corrective feedback was presented for $500 \mathrm{msec}$, followed by a $1,000-\mathrm{msec}$ blank screen and initiation of the next trial.

\section{Results and Theoretical Analyses \\ Matching Task}

To estimate the perceptual representation, we computed the sample orientation and length means (mean vector entries), the sample orientation and length variances, and the sample length-orientation covariance (covariance matrix entries). Because two stimuli were presented simultaneously in the matching task, these variance and covariance estimates actually represent the sum of the standard and comparison stimulus variances and covariances (AlfonsoReese, 1997). Assuming statistical independence between the percepts of the standard and comparison stimuli, we divided each entry in the covariance matrix by $2 .{ }^{4}$ The mean vector and covariance matrix was assumed to represent an estimate of the population parameters for each bivariate normal distribution of perceptual effects. ${ }^{5}$ The 

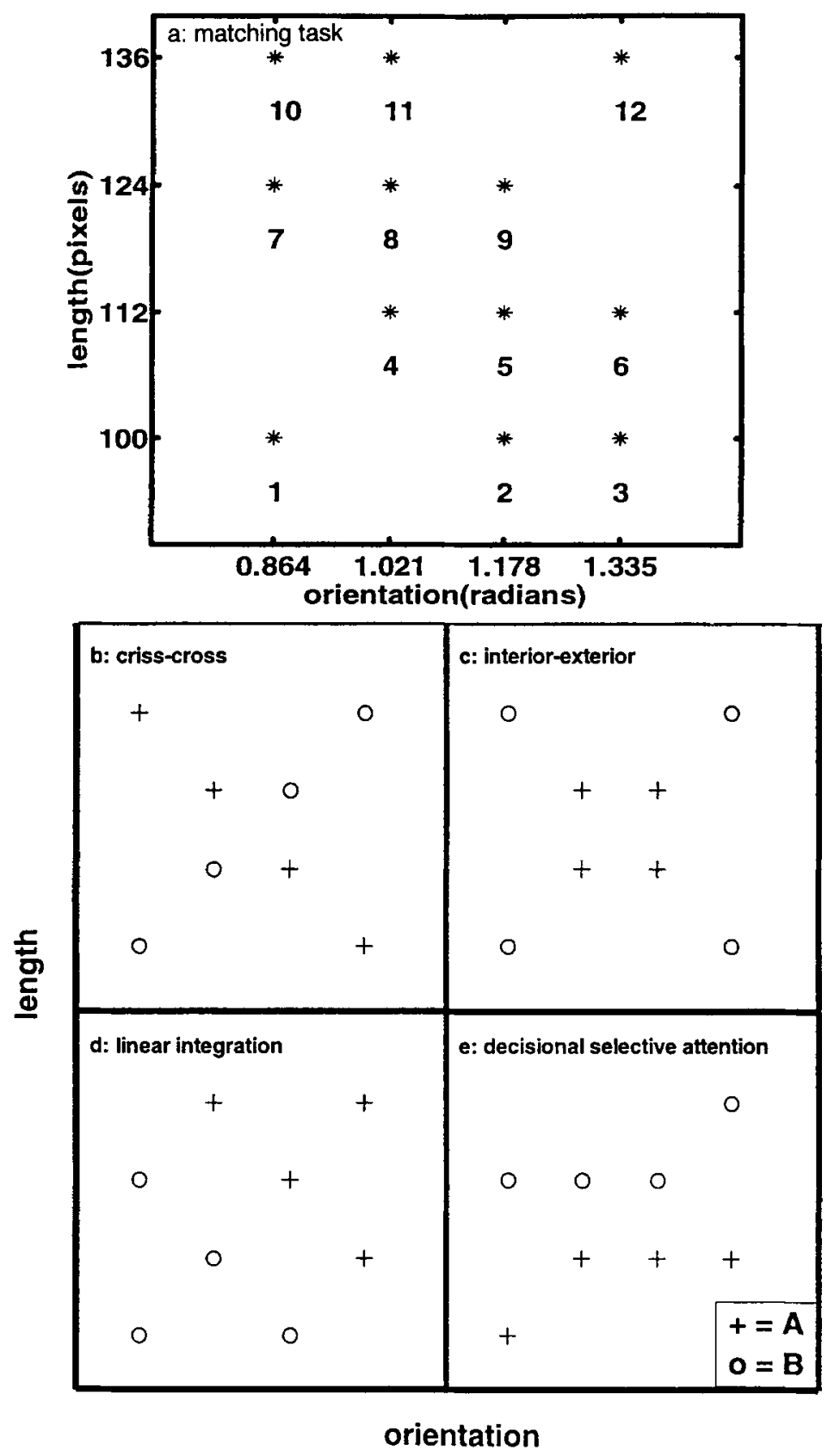

Figure 2. Stimulus structure used in the (a) matching task, and (b) crisscross, (c) interior-exterior, (d) linear integration, and (e) decisional selective attention conditions.

perceptual distribution parameters for each observer are displayed in Table Al.

As suggested by Alfonso-Reese (1997), one advantage of the matching task is that statistical tests of the bivariate normality assumption can be performed. Following Alfonso-Reese (1997), Mardia's (1970, 1974) skewness and kurtosis tests of multivariate normality were performed on all 12 distributions for each observer using a liberal $(\alpha=.05)$ and a conservative $(\alpha=.01)$ signifi- cance value. Assuming a violation of bivariate normality results when both the skewness and kurtosis measures are significant, bivariate normality was rejected for 3,0 , and 7 of the 12 distributions for Observers $1-3$, respectively, using a liberal alpha, and for 2,0 , and 4 of the 12 distributions (in the same order) for a conservative alpha. Thus, the bivariate normality assumption appears reasonable, although some violations were observed, especially for Observer 3. Again following Alfonso-Reese 


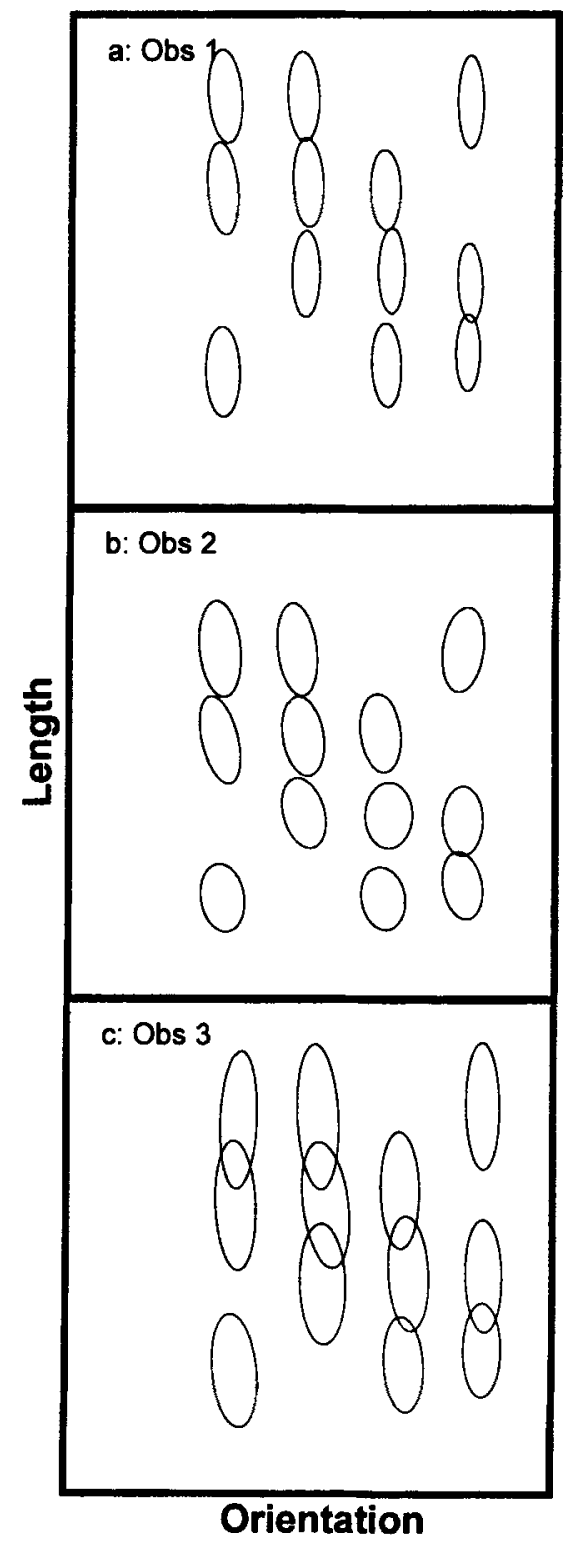

Figure 3. Equal likelihood contours (likelihood $=.003$ ) that describe the perceptual representation estimated from the matching task for (a) Observer 1, (b) Observer 2, and (c) Observer 3.

(1997), we performed tests to determine whether the length and orientation values were statistically independent. When bivariate normality is assumed, statistical independence implies perceptual independence. As a test of statistical independence, likelihood ratio tests (Wilks's $\Lambda$ ) were performed on all 12 distributions for all observers. The hypothesis that length and orientation are statistically independent was rejected for 1,7 , and 2 of the 12 distributions at $\alpha=.05$ and 0,2 , and 1 of 12 distributions at $\alpha=.01$ for Observers $1-3$, respectively. The details of these statistical tests are outlined in the Appendix (Table A2).
Figure 3 displays the equal likelihood contours (for likelihood $=.003$ ) estimated from the matching task for the 12 stimuli for Observers 1-3, respectively. Several aspects of these plots are of interest. First, the representations are nearly perceptually separable, although some violations exist. This was expected since length and orientation are thought to be separable (Garner, 1974; Shepard, 1964). Second, the contours are generally perpendicular to the coordinate axis; this result suggests perceptual independence and converges with the results of our Wilks's $\Lambda$ tests. Third, although not obvious from the plots, variability in perceived length increased monotonically as physical length increased. Specifically, variability in perceived length increased monotonically with physical length at each of the four orientations for Observers 2 and 3 , and at three of four orientations for Observer 1 . This result is predicted from Weber's law. Finally, notice that the resulting perceptual representations are quite similar across observers. The similarities among our equal likelihood contours and to those from previous research (e.g., Ashby \& Lee, 1991) suggest that the matching task provided a reasonable approximation of the true perceptual distributions.

It is important to note that our analyses of these data provide only a rough estimate of perceptual noise. These analyses ignore the possibility that there was noise in the decision process (termed criterial noise). We tried to minimize the effects of criterial noise by (1) using highly trained observers, (2) presenting the stimuli simultaneously, (3) using high-contrast displays, and (4) allowing the observer unlimited time to perform each trial. However, there was probably some criterial noise that is not being accounted for. Even so, one advantage of estimating the perceptual representation from one task (such as the matching task) and then using that representation to predict performance in another task (such as categorization) is that any changes in perceptual and criterial noise across tasks can be estimated. While we would prefer that "true" perceptual noise estimates could be garnered from the matching task, our dual-task approach should allow us to adequately tease apart these two sources of noise. Of course, the strongest test of our claim that the matching task yields reasonable estimates of the perceptual representation will come from actually applying this representation to the categorization data.

\section{Categorization Task}

Accuracy rates. The accuracy rates for the criss-cross, interior-exterior, linear integration, and decisional selective attention conditions for each observer are presented in Table 1 . Table 1 also displays the average accuracy rates across the two nonlinear decision rule conditions (i.e., criss-cross and interior-exterior) and across the two linear decision rule conditions (i.e., linear integration and decisional selective attention). Three results stand out. First, as expected the nonlinear decision rule conditions were more difficult for all observers than the linear decision rule conditions. Second, the interior-exterior condi- 
Table 1

Accuracy Rates for Each Categorization Condition

\begin{tabular}{|c|c|c|c|c|c|c|c|}
\hline \multirow[b]{2}{*}{ Observer } & \multicolumn{4}{|c|}{ Categorization Conditions } & \multirow{2}{*}{$\begin{array}{l}\text { Nonlinear } \\
\text { Decision } \\
\text { Rules }(M)\end{array}$} & \multirow{2}{*}{$\begin{array}{c}\text { Linear } \\
\text { Decision } \\
\text { Rules }(M)\end{array}$} & \multirow{2}{*}{$\begin{array}{c}\text { Overall } \\
M\end{array}$} \\
\hline & $\mathrm{CC}$ & IE & $\mathrm{LI}$ & DSA & & & \\
\hline 1 & 87.00 & 76.38 & 89.75 & 90.50 & 81.69 & 90.13 & 85.91 \\
\hline 2 & 90.00 & 80.25 & 96.75 & 94.75 & 85.13 & 95.75 & 90.44 \\
\hline 3 & 68.13 & 64.63 & 79.63 & 77.00 & 66.38 & 78.32 & 72.35 \\
\hline$M$ & 81.71 & 73.75 & 88.71 & 87.42 & 77.73 & 88.06 & \\
\hline
\end{tabular}

Note-CC, criss-cross; IE, interior-exterior; LI, linear integration; DSA, decisional selective attention.

tion yielded the lowest accuracy. Nosofsky (1986) also found this to be a difficult condition for observers. Finally, decisional selective attention and linear integration accuracy were approximately the same.

Model-based analyses. To facilitate model-based comparisons across length and orientation, a method was needed to equate these two measures. To facilitate this goal, all orientation values (measured in radians) were transformed via a linear transformation with slope $=$ 73.38 and intercept $=34.01$. All model-based analyses were performed at the level of the individual observer. Maddox (1999; see also Maddox \& Ashby, 1998) showed that averaging can alter the structure of the data in such a way that the correct model of individual observer performance might provide a poor account of the aggregate data.

Perceptual representation assumptions. Three different perceptual representations were tested in their ability to predict quantitatively the categorization data. The per- ceptual representation assumptions are outlined in the first column of Table 2 . First, we tested the hypothesis that the perceptual representation, estimated from the matching task, was also the correct perceptual representation for the categorization tasks. Support for this hypothesis validates the matching task as a tool for estimating a perceptual representation. Second, we tested the hypothesis that a perceptually separable representation provides the best account of the categorization data (Ashby \& Lee, 1991; Maddox \& Ashby, 1996). For completeness we also tested the hypothesis that a stimulus invariant perceptual representation provides the best account of the data (Maddox \& Ashby, 1993).

Decision bound assumptions. All models assumed a quadratic decision bound in the criss-cross and interiorexterior conditions, and a linear decision bound in the linear integration and decisional selective attention conditions. ${ }^{6}$ A separate criterial noise parameter was assumed

Table 2

Categorization Model Assumptions

\begin{tabular}{|c|c|c|c|c|}
\hline \multirow{2}{*}{$\begin{array}{c}\text { Representation } \\
\text { Perceptual } \\
\text { Assumptions }\end{array}$} & \multirow[b]{2}{*}{$\begin{array}{c}\text { Decision Bound } \\
\text { Assumptions } \\
\end{array}$} & \multicolumn{3}{|c|}{ Categorization Condition Invariance Assumptions } \\
\hline & & $\begin{array}{l}\text { Condition } \\
\text { Invariance }\end{array}$ & $\begin{array}{c}\text { Linear Invariance/ } \\
\text { Nonlinear Invariance }\end{array}$ & $\begin{array}{c}\text { Decisional Integration/ } \\
\text { Decisional Selective Attention }\end{array}$ \\
\hline $\begin{array}{l}\text { Stimulus Invariance: } \\
\mu_{i} \text { from matching; } \\
\sigma_{o i}=\text { average of } \\
\text { all matching } \sigma_{o i} \\
\sigma_{l i}=\text { average of } \\
\text { all matching } \sigma_{l i} \\
\text { and } \operatorname{cov}_{o l}=0\end{array}$ & $\begin{array}{l}\text { CC and IE: Quadratic } \\
\text { LI and DSA: Linear } \\
\text { ( } 14 \text { parameters); }\end{array}$ & $\begin{array}{l}\text { Perceptual represen- } \\
\text { tation fixed across } \\
\text { conditions with each } \\
\text { orientation variance } \\
\text { modified by a single } \\
\text { additive scalar, and } \\
\text { each length variance } \\
\text { modified by a single } \\
\text { additive scalar } \\
\text { ( } 2 \text { parameters) }\end{array}$ & $\begin{array}{l}\text { CC and IE: } 2 \text { additive } \\
\text { scalars (one for length } \\
\text { and one for orienta- } \\
\text { tion); LI and DSA: } \\
2 \text { additive scalars } \\
\text { (one for length and } \\
\text { one for orientation; } \\
4 \text { parameters) }\end{array}$ & $\begin{array}{l}\text { CC, IE, LI: } 2 \text { additive } \\
\text { scalars (one for length } \\
\text { and one for orientation); } \\
\text { DSA: } 2 \text { additive scalars } \\
\text { (one for length and one } \\
\text { for orientation; } \\
4 \text { parameters) }\end{array}$ \\
\hline $\begin{array}{l}\text { Perceptual Separability: } \\
\mu_{i} \text { and } \Sigma_{i} \text { values } \\
\text { determined by aver- } \\
\text { aging the } \mu_{i} \text { and } \Sigma_{i} \\
\text { values from match- } \\
\text { ing separately for } \\
\text { each level of length } \\
\text { and orientation; } \\
\text { cov }=0 \\
\text { Matching: } \\
\mu_{i} \text { and } \Sigma_{i} \text { from } \\
\text { matching }\end{array}$ & $\begin{array}{l}\text { One criterial noise } \\
\text { parameter per con- } \\
\text { dition ( } 4 \text { parameters) }\end{array}$ & & & \\
\hline
\end{tabular}


for each of the four conditions for all but one model (to be described shortly). Ashby (1992a; see also Ashby \& Maddox, 1993) showed that when the decision bound is quadratic, perceptual and criterial noise are identifiable, but when the decision bound is linear, only the sum of perceptual and criterial noise can be estimated. One of the advantages of modeling categorization data using a perceptual representation estimated from another task is that the perceptual and criterial noise parameters become identifiable, even when the decision bound is assumed to be linear.

Categorization condition invariance assumptions. The simplest assumption, categorization condition invariance, is that the perceptual representation in categorization is unaffected by different categorization decision rules. Thus, the same perceptual representation is assumed for all four categorization conditions. However, we do allow for the possibility that there might be a global change in the perceptual noise that results when the observer shifts from the matching task to the categorization task. This possibility was instantiated by estimating two scalars. One was added to the orientation variance for each stimulus, and the other was added to the length variance for each stimulus. These scalars were allowed to be negative with the constraint that none of the "scaled" variances was negative.

A more general version, linear invariance/nonlinear invariance, assumes that the perceptual representation in categorization is affected by the nature of the categorization decision rule and tests the specific hypothesis that nonlinear decision rules affect the perceptual representation differently from linear decision rules. The fact that categorization performance is strongly affected by the linearity of the categorization rule makes this an important hypothesis to test (see Ashby \& Gott, 1988; Ashby \& Maddox, 1990, 1992; Maddox, Ashby, \& Gottlob, 1998; Maddox \& Bohil, 1998; see also Brehmer, 1987, Hammond \& Summers, 1972, and Mellers, 1980, for similar findings from the multiple cue probability learning literature; but see Medin \& Schwanenflugel, 1981). Specifically, we estimated one pair of additive scalars (one for orientation and one for length) that were applied to the nonlinear categorization conditions (i.e., crisscross and interior-exterior) and another pair of additive scalars that were applied to the linear categorization conditions (i.e., linear integration and decisional selective attention).

Another version, decisional integration invariance/ decisional selective attention invariance, also assumes that the perceptual representation in categorization is affected by the nature of the category decision rule. It tests the hypothesis that categorization decision rules that require decisional integration of (or attention to) both dimensions lead to a different perceptual representation from categorization decision rules that require decisional selective attention to one dimension. Maddox and Ashby (1998; see also Ashby \& Lee, 1991; Maddox \& Ashby, 1996; Maddox et al., 1999) suggested that decisional selective attention may lead to a perceptual form of selec- tive attention that affects the nature of the perceptual representation. To instantiate this, we estimated one pair of additive scalars that were applied to the decisional integration conditions (i.e., criss-cross, interior-exterior, and linear integration) and another pair of additive scalars that were applied only to the decisional selective attention condition. Unfortunately, in this model, the decisional selective attention criterial noise parameter is nonidentifiable with the additive scalars for the decisional selective attention condition. To alleviate this problem, we set the criterial noise parameter to zero and estimated only the scalars. Thus, in this condition the additive scalars represent the sum of the perceptual noise effect and the effects of criterial noise. Fortunately, our focus will be on a comparison of the two scalars with each other, so this nonidentifiability will not adversely affect the comparison.

The factorial combination of three perceptual representation assumptions with three categorization condition invariance assumptions led to the development of nine models, each of which was applied simultaneously to the data from each of the four categorization conditions for each observer. The model parameters were estimated using maximum likelihood procedures (Ashby, 1992b; Wickens, 1982) and the goodness-of-fit statistic (AIC) was

$$
A I C=2 r-2 \ln L,
$$

where $r$ is the number of free parameters and $L$ is the likelihood of the model given the data from the four categorization conditions (Akaike, 1974; Takane \& Shibayama, 1992). ${ }^{7}$ For completeness, we also performed our analyses using sum of squared errors (SSE). We report only the AIC results, but the SSE results led to qualitatively similar conclusions. The total AIC summed across four categorization conditions for each of the nine models is displayed in Table 3 . The three rows for each observer denote the three perceptual representation assumptions. The three columns of data denote the three categorization condition invariance assumptions.

The hypothesis that different categorization decision rules have differential effects on the perceptual repre-

Table 3

Goodness-of-Fit (AIC) Values for the Categorization Models

\begin{tabular}{ccccc}
\hline & & \multicolumn{3}{c}{$\begin{array}{c}\text { Condition Invariance } \\
\text { Assumptions }\end{array}$} \\
\cline { 3 - 5 } Observer & $\begin{array}{c}\text { Perceptual } \\
\text { Representation }\end{array}$ & CI & LI/NLI & DI/DSA \\
\hline 1 & SI & 214.54 & 203.90 & 202.44 \\
& PS & 206.20 & 191.30 & 190.10 \\
& Match & 212.64 & 203.44 & 203.48 \\
2 & SI & 265.42 & 241.42 & 264.70 \\
& PS & 231.28 & 212.12 & 225.24 \\
& Match & 258.74 & 233.66 & 211.62 \\
3 & SI & 230.02 & 234.00 & 231.98 \\
& PS & 229.48 & 233.32 & 231.32 \\
& Match & 228.32 & 231.12 & 235.50 \\
\hline
\end{tabular}

Note-SI, stimulus invariant perceptual representation; PS, perceptual separable representation; Match, matching perceptual representation; CI, categorization condition invariance; LI/NLI, linear invariance/nonlinear invariance; DI/DSA, decisional integration/decisional selective attention. The best-fitting model for each observer is displayed in bold type. 
sentation was supported by the data from Observers 1 and 2 , but was not supported by the data from Observer 3 . One hypothesis states that nonlinear decision rules affect the perceptual representation differently from linear decision rules, whereas the other hypothesis states that decisional integration rules affect the perceptual representation differently from decisional selective attention rules. For Observer 1, both hypotheses received support, although there was a slight advantage for the decisional integration/decisional selective attention hypothesis. For Observer 2, the linear invariance/nonlinear invariance rule was superior for the stimulus invariant and perceptually separable representations, but the decisional integration/decisional selective attention hypothesis was superior for the matching task perceptual representation. These results provide empirical support for Maddox and Ashby's (1998; see also Ashby \& Lee, 1991; Maddox \& Ashby, 1996; Maddox et al., 1999) claim that decisional selective attention might lead to a form of perceptual selective attention. Perceptual selective attention results when the perceptual variance along the decisionally attended dimension is small relative to the perceptual vari- ance along the decisionally unattended dimension. To test this hypothesis we compared the additive scalars for the decisionally attended and unattended dimensions for all 3 observers and the best-fitting perceptual representation (i.e., perceptual separability for Observer 1, and matching for Observers 2 and 3 ). For all 3 observers, the scalar on the attended dimension was much smaller than on the unattended dimension (Observer 1: 4.27 vs. 24.04; Observer 2: 1.78 vs. 88.99 ; Observer $3:-2.75$ vs. 8.90 , for attended and unattended dimension, respectively, in standard deviation units).

In addition, the fit of the best model for each observer was quite good. For example, the percentages of responses accounted for (averaged across four categorization conditions) by the best-fitting model were $99.18,99.25$, and 97.68 for Observers $1-3$, respectively. Even so, the null model (i.e., the model that provided a perfect account of the data) provided a significantly better account of the data than the best-fitting model for all 3 observers (AIC for null model $=174.76,158.12$, and 213.42 for $\mathrm{Ob}$ servers $1-3$, respectively). Clearly there is room for improvement in the model fits. The observed and predicted

Table 4

Observed (Obs) and Predicted (Pred) Probability of

Responding Category " $A$ " for the Best-fitting Model by Condition and Stimulus for Each Observer

\begin{tabular}{|c|c|c|c|c|c|c|c|}
\hline \multirow{2}{*}{$\begin{array}{c}\text { Categorization } \\
\text { Condition }\end{array}$} & \multirow[b]{2}{*}{ Stimulus } & \multicolumn{2}{|c|}{ Observer 1} & \multicolumn{2}{|c|}{ Observer 2} & \multicolumn{2}{|c|}{ Observer 3} \\
\hline & & Obs & Pred & Obs & Pred & Obs & Pred \\
\hline \multirow[t]{8}{*}{$\mathrm{CC}$} & 1 & .010 & .100 & .030 & .000 & .150 & .187 \\
\hline & 3 & .980 & .995 & 1.000 & 1.000 & .800 & .789 \\
\hline & 4 & .220 & .228 & .360 & 247 & .460 & .416 \\
\hline & 5 & .750 & .740 & .910 & .854 & .600 & .592 \\
\hline & 8 & .740 & .749 & .840 & .779 & .550 & .470 \\
\hline & 9 & .270 & .250 & .130 & .185 & .330 & .399 \\
\hline & 10 & 1.000 & .995 & .980 & .999 & .630 & .693 \\
\hline & 12 & .010 & .018 & .010 & .021 & .190 & .178 \\
\hline \multirow[t]{8}{*}{ IE } & 1 & .060 & .055 & .060 & .100 & .200 & .158 \\
\hline & 3 & .020 & .026 & .110 & .108 & .130 & .207 \\
\hline & 4 & .670 & .751 & .900 & .924 & .440 & .482 \\
\hline & 5 & .730 & .639 & .940 & .880 & .590 & .491 \\
\hline & 8 & .920 & .889 & .990 & .945 & .590 & .621 \\
\hline & 9 & .760 & .793 & .830 & .893 & .620 & .619 \\
\hline & 10 & .400 & .381 & .230 & .213 & .460 & .442 \\
\hline & 12 & .150 & .173 & .040 & .050 & .400 & .413 \\
\hline \multirow[t]{8}{*}{$\mathrm{LI}$} & 1 & .000 & .000 & .000 & .000 & .050 & .033 \\
\hline & 2 & .060 & .041 & .030 & .005 & .210 & .270 \\
\hline & 4 & .090 & .098 & .040 & .039 & .290 & .261 \\
\hline & 6 & .740 & .728 & .920 & .943 & .730 & .697 \\
\hline & 7 & .190 & .205 & .090 & .089 & .170 & .232 \\
\hline & 9 & .800 & .861 & 1.000 & .991 & .750 & .694 \\
\hline & 11 & .980 & .937 & .980 & .998 & .700 & .666 \\
\hline & 12 & 1.000 & 1.000 & 1.000 & 1.000 & .910 & .948 \\
\hline \multirow[t]{8}{*}{ DSA } & I & 1.000 & 1.000 & 1.000 & 1.000 & .950 & .972 \\
\hline & 7 & .130 & .906 & .120 & .976 & .390 & .771 \\
\hline & 4 & .940 & .873 & .990 & .950 & .790 & .725 \\
\hline & 8 & .120 & .833 & .080 & .959 & .370 & .734 \\
\hline & 5 & .910 & .175 & .980 & .147 & .830 & .391 \\
\hline & 9 & .120 & .133 & .110 & .116 & .280 & .384 \\
\hline & 6 & .770 & .099 & .930 & .082 & .680 & .304 \\
\hline & 12 & 010 & .000 & .010 & .000 & .050 & .051 \\
\hline
\end{tabular}

Note--CC, criss-cross; IE, interior-exterior; LI, linear integration; DSA, decisional selective attention. 
probabilities of responding " $A$ " for the best-fitting model for each condition and observer are presented in Table 4.

\section{DISCUSSION}

The primary goals of this article were (1) to provide additional validation for the matching task as a method for estimating a perceptual representation and (2) to examine empirically the relation between changing categorization decision rules and the nature of the underlying perceptual representation. The fits of the matching task were quite good, accounting for over $97 \%$ of the variance in each observer's categorization data. This, along with the results of Alfonso-Reese (1997), validates the proposition that the matching task is a good tool for estimating a perceptual representation. Three hypotheses regarding the relation between categorization decision rules and perceptual representation were tested. One hypothesis assumed that the perceptual representation remained unchanged across categorization decision rules. Although rarely stated explicitly, this categorization condition invariance hypothesis is the most common hypothesis made in research. A second hypothesis assumed that nonlinear decision rules have a different effect on the perceptual representation than linear decision rules. A third hypothesis assumed that categorization decision rules that require attention to both stimulus dimensions (termed decisional integration) have a different effect on the perceptual representation than decision rules that require decisional selective attention. The hypothesis that decisional integration affects the perceptual representation differently from decisional selective attention gained support from 2 observers' data. In addition, decisional selective attention led to a smaller perceptual variance along the decisionally attended dimension and to a larger perceptual variance along the decisionally unattended dimension. To our knowledge this is the first study of its kind to contrast several forms of perceptual representation simultaneously with several hypotheses about potential interactions between categorization decision rule and perceptual representation. In addition, these data provide solid evidence that category decision rules, generally assumed to affect only decision processes, can also have a strong effect on perceptual processes and the nature of the perceptual representation.

These results converge with those of Nosofsky's (1986; see also Maddox \& Ashby, 1996) seminal study in showing that selective attention rules often affected the nature of the psychological representation differently from rules that required attention to all stimulus attributes. However, this study goes a step further by teasing apart the separate influences of decisional selective attention tasks on perceptual and decisional processes.

These results also converge with current thinking in the cognitive neurosciences and a recently proposed neuropsychological theory of categorization. Ashby et al. (1998) recently proposed a neuropsychological theory of cate- gorization that postulates a competition between verbal and implicit categorization systems (COVIS). Briefly, the theory proposes two separate and competing categorization systems, one dominated by verbal categorization rules and the other dominated by implicit (nonverbal) categorization rules. The implicit system bases its decision on a perceptual representation computed in some visual area (IT or lower), whereas the verbal system presumably uses a representation from object-based working memory (thought to be in ventrolateral prefrontal cortex; Baddeley, 1995). Current theories of visual selective attention postulate separate attentional effects in low level visual areas, in IT, and in ventrolateral prefrontal cortex (e.g., LaBerge, 1995). Thus, the perceptual representation used by the verbal system receives different (but overlapping) attentional processing than the representation used by the implicit system. In the present study, it is reasonable to suppose that the decisional selective attention condition is dominated by a verbal rule (i.e., if the length is small then respond " $A$ "; if the length is long then respond "B"), whereas the other three conditions are dominated by the implicit system (since no verbal rule exists that could adequately solve these categorization problems). Because the perceptual representations for the verbal and implicit systems receive different (but overlapping) attentional processing, it is possible that the representations would be affected differently. Clearly this reasoning is tentative and requires more rigorous empirical testing. Even so, these data and the present approach provide an interesting crossroads between traditional studies of categorization and the growing interest in cognitive neuroscience.

This study provides important information regarding the potential interaction between changing decision rules and the nature of the perceptual representation in multidimensional perceptual categorization. Even so, it is important not to overgeneralize these results. For example, this study focused exclusively on separable-dimension stimuli. This was a reasonable approach for this initial investigation, but clearly extensions to integral-dimension stimuli are in order. It is possible that interactions between the categorization decision rule and the nature of the perceptual representation will be different for integral- than for separable-dimension stimuli. In addition, the finding that decisional selective attention leads to a different perceptual representation than decisional integration needs to be examined further. Finally, it would be extremely useful to examine empirically the relation between the matching and identification tasks, and to relate each of these to categorization performance.

\section{REFERENCES}

AKAIKE, H. (1974). A new look at the statistical model identification. IEEE Transactions on Automatic Control, 19, 716-723.

AlcoCK, J. (1989). Animal behavior: An evolutionary approach (4th ed.). Sunderland, MA: Sinauer.

ALFONSO-REESE, L. A. (1996). Dynamics of category learning. Unpublished doctoral dissertation, University of California, Santa Barbara. 
ALfonso-ReEse, L. A. (1997). On the dangers of ignoring noise in high-level perception experiments (Technical report). Bloomington: Indiana University.

AshiY, F. G. (1988). Estimating the parameters of multidimensional signal detection theory from simultaneous ratings on separate stimulus components. Perception \& Psychophysics, 44, 195-204.

AshBY, F. G. (1992a). Multidimensional models of categorization. In F. G. Ashby (Ed.), Multidimensional models of perception and cognition (pp. 449-484). Hillsdale, NJ: Erlbaum.

AshBY, F. G. (1992b). Multivariate probability distributions. In F. G. Ashby (Ed.), Multidimensional models of perception and cognition (pp. 1-34). Hillsdale, NJ: Erlbaum.

Ashby, F. G., Alfonso-Reese, L. A., Turken, A. U., \& Waldron, E. M. (1998). A neuropsychological theory of multiple systems in category learning. Psychological Review, 105, 442-481.

Ashby, F. G., \& GoTt, R. E. (1988). Decision rules in the perception and categorization of multidimensional stimuli. Journal of Experimental Psychology: Learning, Memory, \& Cognition, 14, 33-53.

AshBy, F. G., \& LEE, W. W. (1991). Predicting similarity and categorization from identification. Journal of Experimental Psychology: General, 120, 150-172.

AshBY, F. G., \& MADDox, W. T. (1990). Integrating information from separable psychological dimensions. Journal of Experimental Psychology: Human Perception \& Performance, 16, 598-612.

AshBy, F. G., \& MADDOX, W. T. (1991). A response time theory of perceptual independence. In J. P. Doignon \& J. C. Falmagne (Eds.), Mathematical psychology: Current developments (pp. 389-414). New York: Springer-Verlag.

AshBY, F. G., \& MAdDox, W. T. (1992). Complex decision rules in categorization: Contrasting novice and experienced performance. Journal of Experimental Psychology: Human Perception \& Performance, 18, 50-71.

Ashby, F. G., \& MADDox, W. T. (1993). Relations between prototype, exemplar, and decision bound models of categorization. Journal of Mathematical Psychology, 37, 372-400.

AshBy, F. G., \& MadDox, W. T. (1994). A response time theory of perceptual separability and perceptual integrality in speeded classification. Journal of Mathematical Psychology, 33, 423-466.

AshBY, F. G., \& MADDox, W. T. (1998). Stimulus categorization. In M. H. Birnbaum (Ed.), Measurement, judgment, and decision making: Handbook of perception and cognition (pp. 251-301). San Diego: Academic Press.

Ashry, F. G., \& PERrin, N. A. (1988). Toward a unified theory of similarity and recognition. Psychological Review, 95, 124-150.

AshBy, F. G., \& TownSEND, J. T. (1986). Varieties of perceptual independence. Psychological Review, 93, 154-179.

BADDELEY, A. (1995). Working memory. In M. S. Gazzaniga (Ed.), The cognitive neurosciences (pp. 755-764). Cambridge, MA: MIT Press.

Brainard, D. H. (1997). Psychophysics software for use with MATLAB. Spatial Vision, 10, 433-436.

BREHMER, B. (1987). Note on subject's hypothesis in multiple-cue probability learning. Organizational Behavior \& Human Decision Processes, 40, 323-329.

Desimone, R., \& DunCan, J. (1995). Neural mechanisms of selective visual attention. Annual Review of Neuroscience, 18, 193-222.

Desimone, R., \& UNGERLEIDER, L. G. (1989). Neural mechanisms of visual processing in monkeys. In I. Boller \& J. Grafman (Eds.), Handbook of neuropsychology (Vol. 2, pp. 267-299). Amsterdam: Elsevier.

GARNER, W. R. (1974). The processing of information and structure. New York: Wiley.

GarNER, W. R., \& Felfoldy, G. L. (1970). Integrality of stimulus dimensions in various types of information processing. Cognitive Psychology, 1, 225-241.

Geisler, W. S. (1989). Sequential ideal-observer analysis of visual discriminations. Psychological Review, 96, 267-341

Geisler, W. S., \& Albrecht, D. G. (1997). Visual cortex neurons in monkeys and cats: Detection, discrimination, and identification. $V i$ sual Neuroscience, 14, 897-919.

Geisler, W. S. \& CHOU, K. (1996). Separation of low-level and highlevel factors in complex tasks: Visual search. Psychological Review, 102, 356-378.
Goldman-RAKIC, P. S. (1995). Toward a circuit model of working memory and the guidance of voluntary motor action. In J. C. Houk, J. L. Davis, \& D. G. Beiser (Eds.), Models of information processing in the basal ganglia (pp. 131-148). Cambridge, MA: MIT Press, Bradford Books.

GOLDSTONE, R. (1994). Influences of categorization on perceptual discrimination. Journal of Experimental Psychology: General, 123, 178-200.

Green, D. M., \& Swets, J. A. (1967). Signal detection theory and psychophysics. New York: Wiley.

Hammond, K. R., \& Summers, D. A. (1972). Cognitive control. Psychological Review, 79, 58-67.

KADLEC, H., \& HiCKS, C. L. (1998). Invariance of perceptual spaces and perceptual separability of stimulus dimensions. Journal of Experimental Psychology: Human Perception \& Performance, 24, 80-104.

KINGSTON, J., \& MACMILLAN, N. A. (1995). Integrality of nasalization and $F_{1}$ in vowels in isolation and before oral and nasal consonants: $A$ detection-theoretic application of the Garner paradigm. Journal of the Acoustical Society of America, 97, 1261-1285.

Kingston, J., Macmillan, N. A., Dickey, I. W., Thorburn, R., \& BARTELS, C. (1997). Integrality in the perception of tongue root position and voice quality in vowels. Journal of the Acoustical Society of America, 101, 1696-1709.

LABERGE, D. (1995). Computational and anatomical models of selective attention in object identification. In M. S. Gazzaniga (Ed.), The cognitive neurosciences (pp. 649-664). Cambridge, MA: MIT Press.

LABERGE, D. (1997). Attention, awareness, and the triangular circuit. Consciousness \& Cognition, 6, 149-181.

MaCmillan, N. A., \& KingSTon, J. (1995). Integrality, correspondence, and configurality represent different degrees of perceptual interaction, not different types. In C. A. Possamai (Ed.), Fechner Day '95 (pp. 243248). Cassis, France: International Society for Psychophysics.

Macmillan, N. A., \& Ornstein, A. S. (1998). The mean-integral representation of rectangles. Perception \& Psychophysics, 60, 250262.

MadDox, W. T. (1992). Perceptual and decisional separability. In F. G. Ashby (Ed.), Multidimensional models of perception and cognition (pp. 147-180). Hillsdale, NJ: Erlbaum.

MADDOX, W. T. (1999). On the dangers of averaging across observers when comparing decision bound and generalized context models of categorization. Perception \& Psychophysics, 61, 354-374.

Maddox, W. T., \& Ashby, F. G. (1993). Comparing decision bound and exemplar models of categorization. Perception \& Psychophysics, 53, 49-70.

Maddox, W. T., \& Ashby, F. G. (1996). Perceptual separability, decisional separability, and the identification-speeded classification relationship. Journal of Experimental Psychology: Human Perception \& Performance, 22, 795-817.

MadDox, W. T., \& Ashby, F. G. (1998). Selective attention and the formation of linear decision boundaries: Comment on McKinley and Nosofsky (1996). Journal of Experimental Psychology: Human Perception \& Performance, 24, 301-321.

Maddox, W. T., Ashby, F. G., \& Gottlob, L. R. (1998). Response time distributions in multidimensional perceptual categorization. Perception \& Psychophysics, 60, 620-637.

Maddox, W. T., AshBy, F. G., \& Waldron, E. M. (1999). On the functional independence of perceptual and executive attention in perceptual categorization. Manuscript submitted for publication.

Maddox, W. T., \& Bohil, C. J. (1998).Overestimation of base-rate differences in complex perceptual categories. Perception \& Psychophysics, 60, 575-592.

MARDIA, K. V. (1970). Measures of multivariate skewness and kurtosis with applications. Biometrika, 57, 519-530.

MARDIA, K. V. (1974). Applications of some measures of multivariate skewness and kurtosis for testing normality and robustness studies. Sankhya: Series B, 36, 115-128.

Medin, D. L., \& Schaffer, M. M. (1978). Context theory of classification learning. Psychological Review, 85, 207-238.

Medin, D. L., \& SchWANenflugel, P. J. (1981). Linear separability in classification learning. Journal of Experimental Psychology: Human Learning \& Memory, 1, 335-368 
Mellers, B. A. (1980). Configurality in multiple probabilistic cues. American Journal of Psychology, 93, 429-443.

MoRan, J., \& Desimone, R. (1985). Selective attention gates visual processing in the extrastriate cortex. Science, 229, 782-784.

Nosofsky, R. M. (1986). Attention, similarity, and the identificationcategorization relationship. Journal of Experimental Psychology: General, 115, 39-57.

NosOFSKY, R. M. (1998). Selective attention and the formation of linear decision boundaries: Reply to Maddox and Ashby (1998). Journal of Experimental Psychology: Human Perception \& Performance, 24, 322-339.

Olshausen, B. A., Anderson, C. H., \& Van Essen, D. C. (1993). A neurobiological model of visual attention and invariant pattern recognition based on dynamic routing of information. Journal of Neuroscience, 13, 4700-4719.

Perrin, N. A., \& AshBY, F. G. (1991). A test of perceptual independence with dissimilarity data. Applied Psychological Measurement, 15, 79-93.

Posner, M. I., \& Petersen, S. E. (1990). The attention system of the human brain. Annual Review of Neuroscience, 13, 25-42.

ShEPARD, R. N. (1964). Attention and the metric structure of the stimulus space. Journal of Mathematical Psychology, 1, 54-87.

Spitzer, H., Desimone, R., \& Moran, J. (1988). Increased attention enhances both behavioral and neuronal performance. Science, $\mathbf{2 4 0}$, 338-340.

TaKane, Y., \& Shibayama, T. (1992). Structures in stimulus identification data. In F. G. Ashby (Ed.), Multidimensional models of perception and cognition (pp. 335-362). Hillsdale, $\mathrm{NJ}$ : Erlbaum.

Tномаs, R. D. (1995). Gaussian general recognition theory and perceptual independence. Psychological Review, 102, 192-200.

WICKENS, T. D. (1982). Models for behavior: Stochastic processes in psychology. San Francisco: Freeman.

WICKENS, T. D., \& OLZAK, L. A. (1992). Three views of association in concurrent detection ratings. In F. G. Ashby (Ed.), Multidimensional models of perception and cognition (pp. 229-252). Hillsdale, NJ: Erlbaum.

\section{NOTES}

1. Perceptual separability holds when the perceptual effects of a particular component are unaffected by the level of the other component-that is, if the distribution of percepts associated with one component is unaffected by the level of the other component (see Ashby \& Townsend, 1986 for details). In Figure I, Component B is perceptually separable from Component $A$, but Component $A$ is not perceptually separable from Component $B$. A related concept is perceptual independence. Perceptual independence holds for a single stimulus if and only if the perceptual effects for Components A and B are statistically independent (see Ashby, 1988; Ashby \& Maddox, 1991; Ashby \& Townsend, 1986; and Perrin \& Ashby, 1991, for empirical tests of perceptual independence). Perceptual independence is satisfied for all stimuli in Figure 1

2. Alfonso-Reese (1997) used the "noise measurement" technique to study the effects of perceptual noise on categorization performance, but her approach was different from that of the present study and of Ashby and Lee (1991; Maddox \& Ashby, 1996). Alfonso-Reese (1997) collected data from the noise measurement task and used it to estimate the perceptual representation for line stimuli (that differed in length and orientation). These perceptual representations were then used to make qualitative predictions regarding data from two categorization tasks (collected by Ashby et al., 1998) that used several hundred different line stimuli. The present study (similar to Ashby \& Lee, 1991; Maddox \& Ashby, 1996) focused on a quantitative prediction of categorization performance from the perceptual representation estimated from the noise measurement task that used the same stimuli. In addition, the present study (and Ashby \& Lee, 1991; Maddox \& Ashby, 1996) examined the effects of categorization decision rules on the structure of the perceptual representation, an issue not investigated by Alfonso-Reese (1997). Even so, all of these studies supported the claim that perceptual noise can have a powerful influence on categorization performance.

3. Alfonso-Reese (1997) developed an anti-alias routine for Brainard's (1997) Psychophysics Toolbox that minimizes line jaggedness inherent in low-resolution monitors. This routine was not used in the present study, but an examination of the data did not reveal any obvious artifact of jaggedness. Lines that are nearer to horizontal or vertical tend to be more jagged, are probably easier to match, and thus should yield smaller variance estimates. This "jaggedness" hypothesis predicts that the orientation variance estimates should decrease with increasing orientation. Averaged across line length values, the orientation variance estimates generally decreased with increasing orientation, although the decrease was monotonic only for Observer 1. As a test of this hypothesis we conducted one-way within-subjects analyses of variance (ANOVAs) separately for each of the four line length values. The observed significance levels were $.08, .22, .38$, and .29 for line lengths of $100,112,124$, and 136 pixels, respectively. Assuming a lax $\alpha$ level of .10, since each ANOVA contained data from only 3 observers, only data for the line length of 100 pixels supported the "jaggedness" hypothesis. Even so, the anti-alias routine is extremely useful and should be applied whenever possible.

4. The independence assumption is likely incorrect, but in the present situation it should lead to a constant error across stimuli. Since even our simplest modeling application to the categorization data will allow for an additive constant to be applied to the perceptual variances, this "error" should not adversely affect our ability to interpret the data.

5. Two ancillary issues are addressed here. First, one could argue that some sessions should have been considered practice and excluded from the analyses. This is a reasonable argument, but we deemed it important to use all the data to obtain the most stable and accurate estimates of the distribution parameters. This is one reason that we used highly trained observers. Even so, we computed the distribution parameters after excluding Sessions 1-3 and compared these with the parameters estimated from the complete set of data. The mean length estimates differed by $.071, .018$, and $.54 \mathrm{l}$ pixels, and the length variance estimates differed by $.196,1.125$, and .300 pixels squared for Observers $1-3$, respectively. In the same order, the mean orientation estimates differed by $.0006, .0024$, and .0014 radians, and the orientation variance estimates differed by $.00001, .00005$, and .00001 radians squared. Because these difference are quite small, it is unlikely that any of our conclusions would change if we had used Sessions 4-12. Thus, we deem it appropriate to use data from all sessions to derive our distribution estimates. Second, there was some concern that our matching data might have been affected by the magnitude of the initial difference between the standard and comparison stimuli. When the initial difference was large, the observer might get tired of adjusting the comparison stimulus and accept a poor match. This would inflate our estimates of the perceptual variances and would undermine the usefulness of the matching task. To test this hypothesis, we computed the correlation between the error in matching (defined as the Euclidean distance between the standard length and orientation and the "matched" comparison length and orientation) and the initial error (defined as the Euclidean distance between the standard length and orientation, and the initial comparison length and orientation). Correlations were also computed separately for length and orientation. If highly dissimilar standard and comparison values led to large errors, then each correlation should be large and positive. The results were clear. Across the 12 stimuli and 3 observers, the correlations were not significantly different from zero.

6. A series of models were also applied that allowed quadratic decision bounds in all four conditions. In no case did these models provide a superior AIC fit value. In addition, a series of models were applied that assumed optimal decision bounds. These models performed poorly and will not be discussed further.

7. The AIC statistic penalizes a model for extra free parameters in such a way that the smaller the AIC, the closer a model is to the "true model," regardless of the number of free parameters. Thus, to find the best model among a given set of competitors, one simply computes an AIC value for each model and chooses the model associated with the smallest AIC value. A major problem in model fitting is avoiding "local minima." Local minima occur when the parameter estimation algorithm identifies a set of parameters that are "locally" superior (i.e., provide the best fit) but are not "globally" superior. Although one can never be certain that they have obtained the global minima, procedures should be followed to minimize this possibility. Along these lines we tried multiple starting parameter values for all of our fits. We then chose the best of these fits when they did not converge to the same value. In addition, since many of our models were nested, we were able to use the parameters from a more restricted model as starting values for a more general model. These procedures likely improved our changes of identifying the global minima. 
APPENDIX

Table A1

Distribution Parameters From the Matching Task by Observer and Stimulus

\begin{tabular}{|c|c|c|c|c|c|c|}
\hline \multirow[b]{2}{*}{ Observer } & \multirow[b]{2}{*}{ Stimulus No. } & \multicolumn{2}{|c|}{$\begin{array}{l}\text { Orientation } \\
\text { (in radians) }\end{array}$} & \multicolumn{2}{|c|}{$\begin{array}{c}\text { Length } \\
\text { (in pixels) }\end{array}$} & \multirow[b]{2}{*}{ Covariance } \\
\hline & & $M$ & Variance & $M$ & Variance & \\
\hline \multirow[t]{12}{*}{1} & 1 & .886 & .00017 & 99.360 & 6.691 & .0017 \\
\hline & 2 & 1.198 & .00012 & 100.386 & 5.223 & -.0021 \\
\hline & 3 & 1.352 & .00007 & 102.162 & 3.957 & .0005 \\
\hline & 4 & 1.044 & .00011 & 113.301 & 5.740 & .0006 \\
\hline & 5 & 1.207 & .00009 & 113.761 & 5.419 & .0004 \\
\hline & 6 & 1.356 & .00007 & 112.108 & 4.343 & -.0017 \\
\hline & 7 & .884 & .00014 & 125.484 & 6.746 & -.0072 \\
\hline & 8 & 1.048 & .00013 & 126.342 & 6.168 & -.0034 \\
\hline & 9 & 1.194 & .00012 & 125.320 & 4.835 & -.0015 \\
\hline & 10 & .888 & .00017 & 138.492 & 7.103 & -.0063 \\
\hline & 11 & 1.038 & .00014 & 138.455 & 6.182 & -.0026 \\
\hline & 12 & 1.356 & .00008 & 137.748 & 6.077 & .0001 \\
\hline \multirow[t]{12}{*}{2} & 1 & .891 & .00028 & 94.426 & 3.826 & .0048 \\
\hline & 2 & 1.197 & .00028 & 94.410 & 3.180 & -.0040 \\
\hline & 3 & 1.346 & .00022 & 96.317 & 3.622 & -.0060 \\
\hline & 4 & 1.045 & .00029 & 106.560 & 4.046 & -.0100 \\
\hline & 5 & 1.206 & .00034 & 106.307 & 3.677 & .0003 \\
\hline & 6 & 1.346 & .00023 & 105.525 & 3.852 & .0004 \\
\hline & 7 & .885 & .00025 & 116.904 & 6.487 & -.0172 \\
\hline & 8 & 1.043 & .00028 & 117.457 & 5.419 & -.0089 \\
\hline & 9 & 1.190 & .00025 & $117.96 \mathrm{l}$ & 5.144 & -.0060 \\
\hline & 10 & .885 & .00029 & 129.837 & 8.934 & -.0101 \\
\hline & 11 & 1.032 & .00027 & 129.788 & 7.948 & -.0107 \\
\hline & 12 & 1.347 & .00027 & 129.817 & 6.322 & .0084 \\
\hline \multirow[t]{12}{*}{3} & 1 & .921 & .00042 & 97.464 & 14.270 & -.0177 \\
\hline & 2 & 1.242 & .00026 & 98.410 & 8.338 & -.0064 \\
\hline & 3 & 1.389 & .00022 & 100.416 & 7.583 & .0001 \\
\hline & 4 & 1.087 & .00046 & 109.654 & 17.273 & -.0068 \\
\hline & 5 & 1.250 & .00031 & 111.174 & 13.597 & -.0093 \\
\hline & 6 & 1.391 & .00022 & I 10.844 & 11.865 & -.0031 \\
\hline & 7 & .920 & .00035 & 120.681 & 19.840 & -.0111 \\
\hline & 8 & 1.092 & .00053 & 120.788 & 20.495 & -.0314 \\
\hline & 9 & 1.233 & .00028 & 122.914 & 14.433 & -.0037 \\
\hline & 10 & .925 & .00028 & 132.805 & 21.701 & .0095 \\
\hline & 11 & 1.076 & .00042 & 133.258 & 28.430 & -.0181 \\
\hline & 12 & 1.388 & .00019 & 134.848 & 15.957 & -.0025 \\
\hline
\end{tabular}


Table A2

Skewness, Kurtosis, and Independence Tests

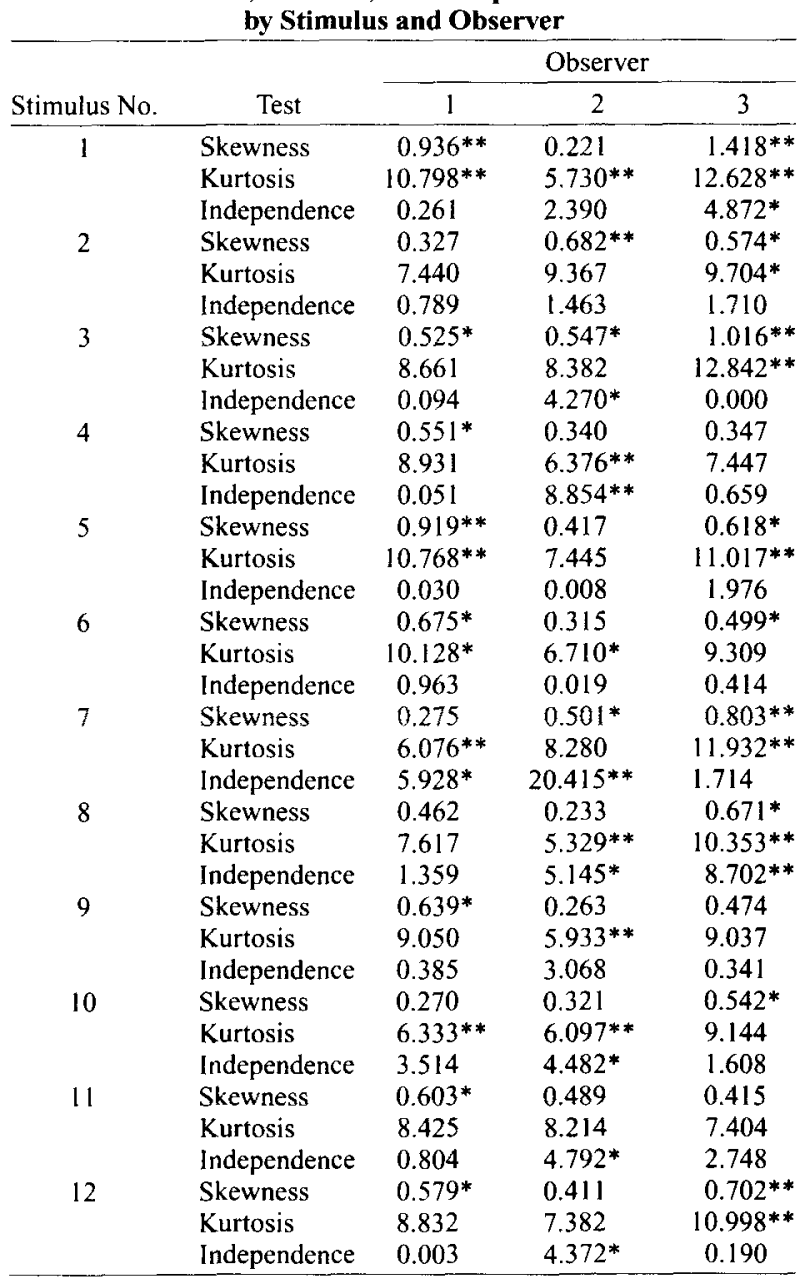

*Significant at $\alpha<.05 . \quad$ **Significant at $\alpha<.01$.

(Manuscript received June 18, 1998;

revision accepted for publication June 29, 1999.) 